\title{
CONE CUBIC CONFIGURATIONS OF A RULED SURFACE*
}

BY

\author{
A. F. CARPENTER
}

\section{INTRODUCTION}

In a series of papers presented to the San Francisco Section of the American Mathematical Societyt during the year 1923, the author has discussed numerous characteristics of certain point and line figurations connected with each line element of the general ruled surface. The definitions and theorems of these papers have been built up by the method of the projective differential geometry of Wilczynski from a few fundamental and well known projective properties of ruled surfaces, chief among which are such notions as the flecnode curve and flecnode surface, the complex curve, the osculating quadric and osculating linear complex.

Before proceeding with the present discussion it will be advisable to restate briefly certain of the definitions and theorems involved.

The flecnode curve $C_{F}$ of a ruled surface $S$ cuts each line element $g$ of $S$ in two points. The planes osculating $C_{F}$ at two such points intersect in a line $h$. For each such line there is determined a second line $h^{\prime}$, the polar reciprocal of $h$ with respect to the linear complex $L$ osculating $S$ along $g$. The three lines $g, h, h^{\prime}$ are in general non-intersecting and hence determine a quadric $Q_{1}$. The complete intersection of $Q_{1}$ and the osculating quadric $Q$ is made up of the element $g$ of $S$ and a space cubic. This curve $C_{F^{*}}$ we call the primary flecnode cubic.

To each osculating plane of $C_{F^{v}}$ there corresponds by means of $L$ a point in that plane. The locus of these points for all the osculating planes of $C_{F}$ is a second space cubic. This curve $C_{F^{\prime}}$ we call the secondary flecnode cubic.

By making use of the complex curve rather than the flecnode curve we arrive at two other cubics called respectively the primary and secondary complex cubics.

Each space cubic determines a linear complex. The complex $L_{1}$ determined by $C_{F^{*}}$ we call the first associated linear complex. It develops that $C_{F^{*}}$ and $C_{F^{\prime}}$ determine the same linear complex. The linear congruence $\boldsymbol{\Gamma}_{\mathbf{1}}$ common to $L$ and $L_{1}$ is called the first associated linear congruence.

* Presented to the Society, San Francisco Section, April 5, 1924.

$\dagger$ April, September, December, 1923. First paper under the title Flecnodal properties of a ruled surface printed in the Tôhoku Mathematical Journal, vol. 23, Nos. 1-2, Aug., 1923. Second paper to appear in the American Journal of Mathematics. 
In a similar manner we obtain from the primary and secondary complex cubics a second associated linear complex and a second associated linear congruence.

As system of reference in the projective differential geometry of ruled surfaces there is frequently used the tetrahedron $P_{y} P_{z} P_{\varrho} P_{\sigma}$. Of the four vertices of this tetrahedron $P_{y}$ and $P_{z}$ are the flecnode points of $l_{y z} \equiv g$, and $P_{\rho}, P_{\sigma}$ are two points, one on each of the flecnode tangents $l_{y \rho}, l_{z \sigma}$, drawn to $S$ at $P_{y}, P_{z}$ respectively. Unit point in this system is so chosen that the coördinates of the four vertices are $P_{y},(1,0,0,0) ; P_{z},(0,1,0,0)$; $P_{\varrho},(0,0,1,0) ; P_{\sigma},(0,0,0,1)$. Of the six edges of the tetrahedron four, namely $l_{y z}, l_{\rho \sigma}, l_{y \sigma}, l_{z e}$, belong to $L$. It follows that the planes which correspond to the points of any one of these lines by means of $L$ constitute a pencil of planes on that line as an axis. There are thus determined four axial pencils.

If, between the points of $l_{y z}$ and $l_{z e}$, there is set up a one-to-one projective correspondence in which to the general point $(\alpha, \beta, 0,0)$ of $l_{y z}$ there corresponds the point $(0, \alpha, \beta, 0)$ of $l_{z \rho}$, then there is likcwise set up a one-to-one projective correspondence between the planes of the two pencils on these two lines. Since the axes of these two projective pencils intersect, the lines of intersection of corresponding pairs of planes must have for their locus a quadric cone $K_{1}$ whose vertex is at $P_{z}$. By making use of the pairs of lines $\left(l_{z \rho}, l_{\rho \sigma}\right),\left(l_{y z}, l_{y \sigma}\right),\left(l_{y \sigma}, l_{\rho \sigma}\right)$, in a similar way we define three other cones $K_{2}, K_{3}, K_{4}$. These four quadric cones we call the complex cones associated with $g$.

Since the pair of lines $\left(l_{y z}, l_{\rho \sigma}\right)$ do not intersect, the two pencils of planes on these lines determine a non-developable quadric. It proves to be in fact the osculating quadric, $Q$. The other pair of non-intersecting lines $\left(l_{z \rho}, l_{y \sigma}\right)$ also determines a quadric, $Q^{\prime} ; Q$ and $Q^{\prime}$ we call the complex quadrics. The equations of the four complex cones and the two complex quadrics, in this system of coördinates, are

$$
\begin{array}{lll}
\left(K_{1}\right) p_{12}^{2} x_{1} x_{3}+p_{21}^{2} x_{4}^{2}=0, & \left(K_{2}\right) p_{12}^{2} x_{1}^{2}+p_{21}^{2} x_{2} x_{4}=0, & \left(K_{3}\right) p_{12}^{2} x_{3}^{2}+p_{21}^{2} x_{2} x_{4}=0 \\
\left(K_{4}\right) p_{12}^{2} x_{1} x_{8}+p_{21}^{2} x_{2}^{2}=0, & (Q) \quad x_{1} x_{4}-x_{2} x_{3}=0, & \left(Q^{\prime}\right) x_{1} x_{2}-x_{3} x_{4}=0
\end{array}
$$

where $p_{12}, p_{21}$ are two of the coefficients of the system of differential equations defining the ruled surface $S$.

The four complex cones $K_{1}, K_{2}, K_{3}, K_{4}$ can be paired in six ways. For each of the four pairs $\left(K_{1} K_{2}\right),\left(K_{3} K_{4}\right),\left(K_{1} K_{3}\right),\left(K_{2} K_{4}\right)$, the complete intersection is composed of a straight line and a space cubic. These four curves $C_{1}, C_{2}, C_{3}, C_{4}$ we call the primary cone cubics associated with $g$. For each of these cubics we indicate below its equations in parametric 
form, the two cones upon which it lies and the line which completes their intersection:

$$
\begin{aligned}
& \left(C_{1}\right) x_{1}=-p_{21}^{2} t, x_{2}=-p_{21}^{2}, \quad x_{3}=p_{12}^{2} t^{3}, x_{4}=p_{12}^{2} t^{2} ; \quad\left(K_{1} K_{2}\right), l_{z q} ; \\
& \left(C_{2}\right) x_{1}=p_{12}^{2} t^{3}, x_{2}=p_{12}^{2} t^{2}, x_{8}=-p_{21}^{2} t, x_{4}=-p_{21}^{2} ; \quad\left(K_{8} K_{4}\right), l_{y \sigma} ; \\
& \left(C_{3}\right) x_{1}=p_{12}^{2} t^{3}, x_{2}=-p_{21}^{2}, \quad x_{8}=-p_{21}^{2} t, x_{4}=p_{12}^{2} t^{2} ; \quad\left(K_{1} K_{3}\right), l_{y z} ; \\
& \left(C_{4}\right) x_{1}=-p_{21}^{2} t, x_{2}=p_{12}^{2} t^{2}, x_{3}=p_{12}^{2} t^{3}, x_{4}=-p_{21}^{2} ; \quad\left(K_{2} K_{4}\right), l_{\rho \sigma} .
\end{aligned}
$$

By making use of their osculating planes and the points corresponding to them by means of $L$, we obtain, from the four cubics $C_{1}, \ldots, C_{4}$, four new cubics $C_{1}^{\prime}, \ldots, C_{4}^{\prime}$, just as the secondary flecnode cubic is obtained from the primary flecnode cubic. These four curves $C_{1}^{\prime}, \ldots, C_{4}^{\prime}$ we call the secondary cone cubics associated with $g$. Their equations in parametric form are

$$
\begin{array}{ll}
\left(C_{1}^{\prime}\right) x_{1}=p_{21}^{3}, & x_{2}=3 p_{12} p_{21}^{2} t, x_{3}=3 p_{12}^{2} p_{21} t^{2}, x_{4}=p_{12}^{3} t^{3} \\
\left(C_{2}^{\prime}\right) x_{1}=3 p_{12}^{2} p_{21} t^{2}, & x_{2}=\quad p_{12}^{8} t^{3}, \quad x_{3}=p_{21}^{3}, \quad x_{4}=3 p_{12} p_{21}^{2} t \\
\left(C_{8}^{\prime}\right) x_{1}=3 p_{12}^{2} p_{21} t^{2}, & x_{2}=-3 p_{12} p_{21}^{2} t, x_{8}=p_{21}^{8}, \quad x_{4}=-p_{12}^{3} ; \\
\left(C_{4}^{\prime}\right) x_{1}=p_{21}^{3}, & x_{2}=-p_{12}^{3} t^{3}, \quad x_{8}=3 p_{12}^{2} p_{21} t^{2}, x_{4}=-3 p_{12} p_{21}^{2} t
\end{array}
$$

The parametric equations of the primary and secondary flecnode cubics, and the equation, in line coordinates, of the linear complex $L_{1}$ which they determine, are respectively

$$
\begin{array}{rll}
\left(C_{F^{*}}\right) & x_{1}=2 t\left(p_{12}^{2} q_{21} t^{2}-p_{21}^{2} q_{12}\right), & x_{2}=2\left(p_{12}^{2} q_{21} t^{2}-p_{21}^{2} q_{12}\right), \\
x_{3}=p_{12} p_{21} t\left(p_{12} t^{2}-p_{21}\right), & x_{4}=p_{12} p_{21}\left(p_{12} t^{2}-p_{21}\right) ; \\
\text { (4) }\left(C_{F^{\prime}}\right) & x_{1}=2 p_{21}\left(3 p_{12}^{2} q_{21} t^{2}+p_{21}^{2} q_{12}\right), & x_{2}=2 p_{12} t\left(p_{12}^{2} q_{21} t^{2}+3 p_{21}^{2} q_{12}\right), \\
& x_{8}=p_{12} p_{21}^{2}\left(3 p_{12} t^{2}+p_{21}\right), & x_{4}=p_{12}^{2} p_{21} t\left(p_{12} t^{2}+3 p_{21}\right) ; \\
\left(L_{1}\right) 2 p_{12} p_{21} \omega_{12}-\left(p_{12} q_{21}+3 p_{21} q_{12}\right) \omega_{14}+\left(3 p_{12} q_{21}+p_{21} q_{12}\right) \omega_{23}+8 q_{12} q_{21} \omega_{34}=0 ;
\end{array}
$$

where $q_{12}, q_{\mathbf{2 1}}$, are another pair of coefficients of the system of differential equations defining $S$.

The equation of the quadric $Q_{1}$ is

$$
\left(Q_{1}\right) \quad p_{12}^{2} p_{21} x_{1} x_{8}-p_{12} p_{21}^{2} x_{2} x_{4}-2 p_{12}^{2} q_{21} x_{3}^{2}+2 p_{21}^{2} q_{12} x_{4}^{2}=0 \text {. }
$$

If we identify the parameters in the equations of the ten cubics listed above we thereby set up a point correspondence between these curves. But this correspondence is not arbitrary. That between $C_{F}$ and $C_{F^{\prime \prime}}$ is 
indeed the one resulting from the definition of $C_{F^{\prime \prime}}$. The projective transformation*

$$
\begin{array}{ll}
\bar{x}_{1}=-p_{21} x_{1}+2 q_{21} x_{3}, & \bar{x}_{2}=-p_{21} x_{2}+2 q_{91} x_{4}, \\
\bar{x}_{3}=p_{12} x_{1}-2 q_{12} x_{3}, & \bar{x}_{4}=p_{12} x_{2}-2 q_{12} x_{4}
\end{array}
$$

carries $C_{F^{\prime}}$ into $C_{1}$ and $C_{F^{\prime \prime}}$ into $C_{1}^{\prime}$. An examination of equations (2) shows that the four curves $C_{1}, \ldots, C_{4}$ are projectively equivalent, the projectivities which carry $C_{1}$ into $C_{2}, C_{3}, C_{4}$ being respectively

$$
\begin{aligned}
& \bar{x}_{1}=x_{3}, \quad \bar{x}_{2}=x_{4}, \quad \bar{x}_{3}=x_{1}, \quad \bar{x}_{4}=x_{2} ; \\
& \bar{x}_{1}=x_{8}, \quad \bar{x}_{2}=x_{2}, \quad \bar{x}_{8}=x_{1}, \quad \bar{x}_{4}=x_{4} ; \\
& \bar{x}_{1}=x_{1}, \quad \bar{x}_{2}=x_{4}, \quad \bar{x}_{8}=x_{3}, \quad \bar{x}_{4}=x_{2} .
\end{aligned}
$$

Similarly for the curves $C_{1}^{\prime}, \ldots, C_{4}^{\prime}$, but with a different set of projectivities. The point correspondence between the five curves $C_{F^{\prime}}, C_{1}, C_{\mathbf{2}}$, $C_{3}, C_{4}$, as well as that between the five curves $C_{F^{\prime \prime}}, C_{1}^{\prime}, C_{2}^{\prime}, C_{3}^{\prime}, C_{4}^{\prime}$, is therefore projective in nature, while that between the pairs $C_{j}, C_{j}^{\prime}$ $(j=1, \ldots, 4)$, is exactly that determined by the complex $L$ between $C_{F^{\prime}}$ and $C_{F^{\prime \prime}}$.

From the general theoryt a fundamental system of simultaneous solutions $y_{k}(x), z_{k}(x)(k=1, \ldots, 4)$ of the system of equations defining $S$ determines two directrix curves $C_{y}, C_{z}$ of $S$. Corresponding points $P_{y}, P_{z}$ of these curves have the homogeneous coördinates $\left(y_{1}, y_{2}, y_{3}, y_{4}\right),\left(z_{1}, z_{2}, z_{3}, z_{4}\right)$ and lie upon the same generator $g$ of $S$. For our choice of a coordinate system, as we have seen, $y_{1}=1, y_{2}=y_{3}=y_{4}=0 ; z_{2}=1, z_{1}=z_{3}=z_{4}=0$; while $\varrho_{3}=1, \varrho_{1}=\varrho_{2}=\varrho_{4}=0 ; \sigma_{4}=1, \sigma_{1}=\sigma_{2}=\sigma_{3}=0$; where

$$
\varrho=2 \frac{d y}{d x}+p_{12} z, \quad \sigma=2 \frac{d z}{d x}+p_{21} y .
$$

For the general point $P_{x} ;(a, b, c, d)$, the coorrdinates are given by the expression

$$
x_{k}=a y_{k}+b z_{k}+c \varrho_{k}+d \sigma_{k} \quad(k=1, \cdots, 4),
$$

the subscript $k$ ordinarily being omitted.

${ }^{*} p_{12}, p_{21}, q_{12}, q_{21}$ are functions of a parameter $x$ which is constant so long as we are considering a single line element $g$ of $S$. Hence this transformation is projective for all configurations associated with $g$.

† Projective Differential Geometry of Curves and Ruled Surfaces, Wilczynski; B. G. Teubner, 1906, pp. 129, 130. Hereafter referred to as Proj. Dif. Geom. 
It is the purpose of this paper to characterize with reasonable completeness the configurations formed by the ten curves $C_{F^{\prime}}, C_{F^{\prime \prime}} ; C_{1}, \ldots, C_{4} ; C_{1}^{\prime}, \ldots, C_{4}^{\prime}$, to define and discuss two additional sets of cubics, and to suggest further problems for investigation.

\section{The FIRST AND SECOND CONE RAYS}

There are four pencils of quadrics determined by the pairs of cones $\left(K_{1} K_{2}\right)$, $\left(K_{3} K_{4}\right),\left(K_{1} K_{3}\right),\left(K_{2} K_{4}\right)$, namely

$$
\begin{aligned}
& h\left(p_{12}^{2} x_{1} x_{3}+p_{21}^{2} x_{4}^{2}\right)+k\left(p_{12}^{2} x_{1}^{2}+p_{21}^{2} x_{2} x_{4}\right)=0 \\
& h\left(p_{12}^{2} x_{3}^{2}+p_{21}^{2} x_{2} x_{4}\right)+k\left(p_{12}^{2} x_{1} x_{8}+p_{21}^{2} x_{2}^{2}\right)=0 \\
& h\left(p_{12}^{2} x_{1} x_{8}+p_{21}^{2} x_{4}^{2}\right)+k\left(p_{12}^{2} x_{3}^{2}+p_{21}^{2} x_{2} x_{4}\right)=0 \\
& h\left(p_{12}^{2} x_{1}^{2}+p_{21}^{2} x_{2} x_{4}\right)+k\left(p_{12}^{2} x_{1} x_{3}+p_{21}^{2} x_{2}^{2}\right)=0
\end{aligned}
$$

By using the same parameters in each of the four equations (7) we have set up an arbitrary correspondence between the quadrics of these four pencils. We do this as a matter of convenience.

$C_{1}$ lies upon each of the quadrics of $\left(7_{1}\right)$. Since $C_{1}$ also lies upon $Q$ each of these quadrics $\left(7_{1}\right)$ must have in common with $Q$ one, and only one, straight line. It proves to be the line joining the point $h y-k \varrho$ on $l_{y \rho}$ to the point $h z-k \sigma$ on $l_{z \sigma}$. As we pass from surface to surface of the pencil $\left(7_{1}\right)$, this line coincides successively with all the rulings of $Q$.

$C_{2}$ lies upon each of the quadrics of $\left(7_{2}\right)$. Since $C_{2}$ also lies upon $Q$, each of these quadrics $\left(7_{2}\right)$ must have in common with $Q$ one, and only one, straight line. It also proves to be the line determined by the points $h y-k \varrho$ and $h z-k \sigma$, so that corresponding quadrics of the pencils $\left(7_{1}\right)$ and $\left(7_{2}\right)$ cut $Q$ in the same line.

The cubics $C_{3}, C_{4}$ are seen by inspection to lie upon the quadric $Q^{\prime}$. But $C_{3}$ lies upon each quadric of the pencil $\left(7_{3}\right)$ and $C_{4}$ upon each quadric of the pencil $\left(7_{4}\right)$. Each quadric of $\left(7_{3}\right)$, as also each of $\left(7_{4}\right)$, must therefore have one, and only one, straight line in common with $Q^{\prime}$. This line is seen to be that which is determined by the two points $k y-h \varrho, h z-k \sigma$ on $l_{y \rho}, l_{z \sigma}$, respectively. Corresponding pairs of quadrics of the two pencils $\left(7_{3}\right),\left(7_{4}\right)$ have this line in common with $Q^{\prime}$. As we pass from surface to surface of $\left(7_{3}\right)$ or $\left(7_{4}\right)$, this line coincides successively with all of the rulings of $Q^{\prime}$.

If we write for the general point on the first of these lines the expression

$$
f(h y-k \varrho)+g(h z-k \sigma),
$$


and, for the general point on the second, the expression

$$
m(k y-h \varrho)+n(h z-k \sigma),
$$

then it follows that these two points will coincide if and only if

$$
\frac{h}{k}=\frac{k}{h}, \text { that is, if } h= \pm k .
$$

One may thus in two ways choose a set of four quadrics, one from each pencil, such that the four will have a line in common and this line will at the same time lie upon both the complex quadrics. The points in which these two lines cut the flecnode tangents $l_{y \rho}, l_{z \sigma}$ will engage our attention again. They are given by the expressions

$$
\alpha=y-\varrho, \quad \beta=z-\sigma ; \quad \gamma=y+\varrho, \quad \delta=z+\sigma .
$$

We note that $P_{\alpha}, P_{\gamma}$ are harmonic conjugates with respect to $P_{y}, P_{\varrho}$, as are also $P_{\beta}, P_{\delta}$, with respect to $P_{z}, P_{\sigma}$.

The cones $K_{1}$ and $K_{2}$ have $C_{1}$ and $l_{z \rho}$ as their complete intersection. The line $l_{z \rho}$ is tangent to $K_{3}$ at $P_{z}$ but is not an element of $K_{3}$. If we substitute from $\left(C_{1}\right)$ of (2) into $\left(K_{3}\right)$ of (1) we find for $t$ the six values

$$
0, \quad 0, \quad p_{21} / p_{12}, \quad-p_{21} / p_{12}, \quad p_{21} i / p_{12}, \quad-p_{21} i / p_{12} .
$$

The first two of these correspond to the point $P_{z}$ and the remaining four, by means of $\left(C_{1}\right)$, give respectively the four points $P_{\varphi}, P_{\psi}, P_{\eta}, P_{\xi}$, where

$$
\begin{aligned}
& \varphi=p_{21} y+p_{12} z-p_{21} \varrho-p_{12} \sigma=p_{21} \alpha+p_{12} \beta, \\
& \psi=p_{21} y-p_{12} z-p_{21} \varrho+p_{12} \sigma=p_{21} \alpha-p_{12} \beta, \\
& \eta=p_{21} i y+p_{12} z+p_{21} i \varrho+p_{12} \sigma=p_{21} i \gamma+p_{18} \delta, \\
& \zeta=p_{21} i y-p_{12} z+p_{21} i \varrho-p_{12} \sigma=p_{21} i \gamma-p_{12} \delta .
\end{aligned}
$$

From $\left(K_{4}\right)$ of (1), the point $P_{z}$ is not on $K_{4}$, but the four points $P_{\varphi}$, $P_{\psi}, P_{\eta}, P_{\zeta}$ are. Moreover, (9) shows that these four points are also on $Q$ and $Q^{\prime}$. Two of them, $P_{\varphi}, P_{\psi}$, are in fact harmonic conjugates with respect to $P_{\alpha}, P_{\beta}$, and the other two, $P_{\eta}, P_{\xi}$, harmonic conjugates with respect to $P_{\gamma}, P_{\delta}$. These four points $P_{\varphi}, P_{\psi}, P_{\eta}, P_{\zeta}$, are the only ones common to all four cones $K_{1}, \cdots, K_{4}$. Since they may be thought of as determining the two lines $l_{\alpha \beta}$, $l_{\gamma \delta}$, we shall speak of these two lines as the first and second cone rays associated with $g$. 
The four points $P_{\varphi}, P_{\psi}, P_{\eta}, P_{\zeta}$ have already been shown to lie on $C_{1}$. By introducing the values $t= \pm p_{21} / p_{12}, \pm p_{21} i / p_{12}$ into $\left(C_{8}\right),\left(C_{3}\right),\left(C_{4}\right)$ of (2) it results that all four curves pass through these four points and that in the point correspondence existing between the four curves, these four points are self-corresponding. It should be noted that $P_{\varphi}$, thought of as being on $C_{1}, C_{2}$, corresponds to $P_{\psi}$, thought of as being on $C_{3}, C_{4}$, and conversely, while $P_{\eta}$ counts for a complete set of four corresponding points, as does also $P_{\zeta}$.

All four points cannot be real at the same time, the two on the second cone ray, $l_{\gamma \delta}$, being imaginary if those on the first cone ray, $l_{\alpha \beta}$, are real, and conversely. It may happen that none of the four are real. We shall speak of these four points as the focal points of the primary cone cubics.

If $(f, g, h, k)$ be any point, then the three points whose coordinates are $(h, k, f, g),(h, g, f, k),(f, k, h, g)$, as well as the three points whose coordinates are $(h, k, f, g),(h,-g, f,-k),(f,-k, h,-g)$, determine a plane on which the first point lies. It follows from equations (5) that if $P_{1}, P_{2}, P_{3}, P_{4}$ are a set of four corresponding points, one on each of the curves $C_{1}, C_{2}, C_{3}, C_{4}$, then $P_{1}, P_{2}, P_{3}, P_{4}$ are coplanar. Likewise it follows that if $P_{1}^{\prime}, P_{2}^{\prime}, P_{3}^{\prime}, P_{4}^{\prime}$ are a set of four corresponding points, one on each of the curves $C_{1}^{\prime}, C_{2}^{\prime}, C_{3}^{\prime}, C_{4}^{\prime}$, then $P_{1}^{\prime}, P_{2}^{\prime}, P_{3}^{\prime}, P_{4}^{\prime}$ are coplanar. The equations of the planes $p$ and $p^{\prime}$ containing these two sets of points are easily found. For the first set we find the equation

$$
x_{1}+x_{8}-t\left(x_{2}+x_{4}\right)=0 \text {, }
$$

and for the second set, the equation

where

$$
\begin{gathered}
p_{12} \times t\left(x_{1}-x_{3}\right)-p_{21} \lambda\left(x_{2}-x_{4}\right)=0, \\
x=p_{12}^{2} t^{2}-3 p_{21}^{2}, \quad \lambda=3 p_{12}^{2} t^{2}-p_{21}^{2} .
\end{gathered}
$$

The points $\alpha=y-\varrho, \beta=z-\sigma$ are on the first of these two planes and the points $\gamma=y+\varrho, \delta=z+\sigma$ are on the second. It follows that equations (10) and (11) define two pencils of planes whose axes are the first and second cone rays, $t$ being the parameter of the pencil in each case. In brief, the projectivity existing between the points of the four primary (secondary) cone cubics is such that corresponding points lie by fours in the planes of an axial pencil whose axis is the first (second) cone ray. We note from (4), (10) and (11) that the point on the primary flecnode cubic which corresponds to the set of points $P_{1}, \cdots, P_{4}$ lies on their plane, but that the point of the secondary flecnode cubic which corresponds to the set $P_{1}^{\prime}, \ldots, P_{4}^{\prime}$ lies on their plane only if $p_{12}-2 q_{12}=p_{21}-2 q_{21}$. 


\section{Perspectivities of the CONE cubics}

Since the cubics $C_{1}$ and $C_{2}$ lie on $Q$ while the cubics $C_{3}$ and $C_{4}$ lie on $Q^{\prime}$, it results that of the four points $P_{1}, P_{2}, P_{3}, P_{4}$, in which these four curves cut the plane $p, P_{1}$ and $P_{2}$ lie on $Q$ and $P_{3}$ and $P_{4}$ upon $Q^{\prime}$. Since $l_{\alpha \beta}$ lies on both $Q$ and $Q^{\prime}$, the plane $p$ through this line must be a tangent plane to both quadrics. Its points of tangency with $Q$ and $Q^{\prime}$ are given by the respective expressions

$$
\tau=t \alpha+\beta, \quad \boldsymbol{\tau}^{\prime}=-t \alpha+\beta .
$$

These points are harmonic conjugates with respect to $P_{\alpha}$ and $P_{\beta}$. We note that the points $P_{1}, P_{2}$ are not on $l_{\alpha \beta}$. They must therefore lie upon the second line which $p$ has in common with $Q$, so that $P_{1}, P_{2}$ and $P_{\tau}$ are collinear. A similar argument leads to the conclusion that $P_{3}, P_{4}$ and $P_{\tau^{\prime}}$ are collinear. We find further that $P_{1}, P_{3}$ and $P_{\alpha}$ are collinear as are also the sets $P_{2}, P_{1}, P_{\alpha} ; P_{1}, P_{4}, P_{\beta} ; P_{2}, P_{3}, P_{\beta}$.

The lines $P_{1} P_{2}$ and $P_{3} P_{4}$, lying in plane $p$, intersect. But $P_{1} P_{2}$ is a ruling of $Q$ and $P_{3} P_{4}$ a ruling of $Q^{\prime}$. Their point of intersection must therefore be a point of the intersection of $Q$ and $Q^{\prime}$. The complete intersection of these two quadrics consists of the two flecnode tangents $l_{y \varrho}, l_{z \sigma}$, and the two cone rays $l_{\alpha \beta}, l_{\gamma \delta}$. Moreover the flecnode tangents belong to one regulus and the cone rays to the other regulus, on each quadric. Since $P_{1} P_{2}$ and $P_{3} P_{4}$ intersect $l_{\alpha \beta}$ in distinct points, their point of intersection must be on $l_{\gamma \delta}$. It is indeed the point given by the expression

$$
\boldsymbol{\theta}=t \gamma+\delta .
$$

To sum up, we find that the complete quadrangle whose vertices $P_{1}, \ldots, P_{4}$ are any set of corresponding primary cone cubic points lies on a plane which is at the same time tangent to both of the complex quadrics $Q$ and $Q^{\prime}$. Of its six sides, one, $P_{1} P_{2}$, lies on $Q$ and another, $P_{3} P_{4}$, lies on $Q^{\prime}$. Of its diagonal points, two, $P_{\alpha}, P_{\beta}$, lie upon the first cone ray and are the same for all such quadrangles, while the third lies upon the second cone ray.

That plane of the pencil (10) which is tangent to $Q$ at the point $P_{\tau_{1}}$, where $\tau_{1}=t_{1} \alpha+\beta$, is given by the equation

$$
x_{1}+x_{3}-t_{1}\left(x_{2}+x_{4}\right)=0 .
$$

The tangent plane to $Q^{\prime}$ at this same point has for its equation

$$
x_{1}+x_{3}+t_{1}\left(x_{2}+x_{4}\right)=0 .
$$


But the plane $\left(10_{1}\right)$ is tangent to $Q^{\prime}$ at $P_{\tau_{-1}}$, where $\tau_{-1}=-t_{1} \alpha+\beta$, and the plane $\left(10_{-1}\right)$ is tangent to $Q$ at this same point.

Let us think of the planes of the pencil (10) as paired in this way, the two planes of such a pair being indicated by $p_{1}$ and $p_{-1}$ and the quadrangles in these planes by $\left(P_{1} P_{2} P_{3} P_{4}\right)$ and $\left(P_{-1} P_{-2} P_{-3} P_{-4}\right)$. Then it is seen that in either of the two orders

$$
\left(P_{1} P_{2} P_{3} P_{4}\right) \sim\left(P_{-3} P_{-4} P_{-1} P_{-2}\right) \text { or }\left(P_{1} P_{2} P_{8} P_{4}\right) \sim\left(P_{-4} P_{-8} P_{-2} P_{-1}\right),
$$

we can determine a correspondence between these two quadrangles such that corresponding pairs of lines intersect in $l_{\alpha \beta}$. We find indeed that the lines $P_{1} P_{3}, P_{2} P_{4}, P_{-1} P_{-8}, P_{-2} P_{-4}$ intersect in $P_{\alpha}$, the lines $P_{1} P_{4}$, $P_{2} P_{3}, P_{-1} P_{-4}, P_{-2} P_{-3}$ intersect in $P_{\beta}$, the lines $P_{1} P_{2}, P_{-8} P_{-4}$, in the point $P_{\tau}$ and the lines $P_{3} P_{4}, P_{-1} P_{-2}$, in the point $P_{\tau^{\prime}}$.

It follows that in two ways these two quadrangles are in perspective from a point. It can be easily verified that the four lines $P_{1} P_{-8}, P_{2} P_{-4}$, $P_{3} P_{-1}, P_{4} P_{-2}$ pass through the point $P_{\gamma}$, while the lines $P_{1} P_{-4}, P_{2} P_{-3}$, $P_{3} P_{-2}, P_{4} P_{-1}$ pass through the point $P_{\delta}$.

Without further discussion we remark that if the planes of the pencil on the second cone ray $l_{\gamma \delta}$ are paired as above, so that of the two in each pair, the first, $q_{1}$,

$$
x_{1}-x_{3}-t_{1}\left(x_{2}-x_{4}\right)=0,
$$

is tangent to $Q$ at $\theta_{1}=t_{1} \gamma+\delta$, while the second, $q_{-1}$,

$$
x_{1}-x_{3}+t_{1}\left(x_{2}-x_{4}\right)=0,
$$

is tangent to $Q^{\prime}$ at the same point, then the quadrangle $\left(P_{1} P_{2} P_{-3} P_{-4}\right)$ lies in the first of these planes, the quadrangle $\left(P_{3} P_{4} P_{-1} P_{-2}\right)$ lies in the second, and these two quadrangles are in perspective from the two points $P_{\alpha}, P_{\beta}$, the four lines $P_{1} P_{3}, P_{2} P_{4}, P_{-3} P_{-1}, P_{-4} P_{-2}$ passing through $P_{\alpha}$ and the four lines $P_{1} P_{4}, P_{2} P_{3}, P_{-3} P_{-2}, P_{-4} P_{-1}$ through $P_{\beta}$.

To recapitulate: let those points of the first and second cone rays $l_{\alpha \beta}, l_{\gamma \delta}$ correspond which lie upon the same line of that regulus of $Q$ to which the flecnode tangents belong. At each of such a pair of corresponding points $P_{\tau}, P_{\theta}$, construct the tangent planes to $Q$ and $Q^{\prime}$. Of these four planes, two, $p_{1}, p_{-1}$, are on $l_{\alpha \beta}$, and two, $q_{1}, q_{-1}$, are on $l_{\gamma \delta}$. Of the total of $3.4 \cdot 4=48$ points of intersection of these four planes with the four primary cone cubics, 32 coincide by eights in the four focal points $P_{\varphi}, P_{\psi}, P_{\eta}, P_{\zeta}$. The remaining 16 points coincide by twos. 
Of these eight distinct points four lie in each of the planes $p_{1}, p_{-1}, q_{1}$, $q_{-1}$, in such a way that no plane contains more than one point from each cone cubic. Consider the points $P_{\alpha}, P_{\beta}$, and $P_{\gamma}, P_{\delta}$, in which $l_{\alpha \beta}$ and $l_{\gamma \delta}$ are cut by $l_{\alpha \gamma}$ and $l_{\beta \delta}$. The lines joining either $P_{\gamma}$ or $P_{\delta}$ to the four cone cubic points in either $p_{1}$ or $p_{-1}$ pass through the cone cubic points in the other of these two planes, and the lines joining either $P_{\alpha}$ or $P_{\beta}$ to the four cone cubic points in either $q_{1}$ or $q_{-1}$ pass through the four cone cubic points in the other of these two planes.

We have already seen that, for each set of corresponding points $P_{1}$, $P_{2}, P_{3}, P_{4}$, lying in the plane $p_{1}$, there exists a set of corresponding secondary cone cubic points $P_{1}^{\prime}, P_{2}^{\prime}, P_{8}^{\prime}, P_{4}^{\prime}$, lying in the plane $p_{1}^{\prime}$,

$$
p_{12} x_{1} t_{1}\left(x_{1}-x_{3}\right)-p_{21} \lambda_{1}\left(x_{2}-x_{4}\right)=0 \text {. }
$$

Similarly, for the set of points $P_{-1}, P_{-2}, P_{-3}, P_{-4}$, lying in the plane $p_{-1}$, there is a set $P_{-1}^{\prime}, P_{-2}^{\prime}, P_{-3}^{\prime}, P_{-4}^{\prime}$, lying in the plane $p_{-1}^{\prime}$,

$$
p_{12} x_{1} t_{1}\left(x_{1}-x_{3}\right)+p_{21} \lambda_{1}\left(x_{2}-x_{4}\right)=0 \text {. }
$$

For these two quadrangles lying in the planes $p_{1}^{\prime}, p_{-1}^{\prime}$, it also holds that in either of two orders,

$$
\left(P_{1}^{\prime} P_{2}^{\prime} P_{3}^{\prime} P_{4}^{\prime}\right) \sim\left(P_{-3}^{\prime} P_{-4}^{\prime} P_{-1}^{\prime} P_{-2}^{\prime}\right) \quad \text { or }\left(P_{1}^{\prime} P_{2}^{\prime} P_{3}^{\prime} P_{4}^{\prime}\right) \sim\left(P_{-4}^{\prime} P_{-3}^{\prime} P_{-2}^{\prime} P_{-1}^{\prime}\right) \text {, }
$$

we can determine a correspondence between them such that corresponding pairs of lines intersect on $l_{y \delta}$. We find that the lines $P_{1}^{\prime} P_{3}^{\prime}, P_{2}^{\prime} P_{4}^{\prime}$, $P_{-1}^{\prime} P_{-3}^{\prime}, P_{-2}^{\prime} P_{-4}^{\prime}$ intersect in $P_{y}$, the lines $P_{1}^{\prime} P_{4}^{\prime}, P_{2}^{\prime} P_{8}^{\prime}, P_{-1}^{\prime} P_{-4}^{\prime}$, $P_{-2}^{\prime} P_{-8}^{\prime}$, in $P_{\delta}$, the lines $P_{1}^{\prime} P_{2}^{\prime}, P_{-3}^{\prime} P_{-4}^{\prime}$, in $P_{\xi}$, and the lines $P_{3}^{\prime} P_{4}^{\prime}$, $P_{-1}^{\prime} P_{-2}^{\prime}$, in $P_{\xi^{\prime}}$, where

$$
\begin{array}{ll}
\xi=p_{21} \nu \gamma+p_{12} \mu t \delta, & \xi^{\prime}=p_{21} \nu \gamma-p_{12} \mu t \delta, \\
\nu=3 p_{12}^{2} t^{2}+p_{21}^{2}, & \mu=p_{12}^{2} t^{2}+3 p_{21}^{2} .
\end{array}
$$

It follows that in two ways these two quadrangles are in perspective from a point. Indeed the lines $P_{1}^{\prime} P_{-8}^{\prime}, P_{2}^{\prime} P_{-4}^{\prime}, P_{3}^{\prime} P_{-1}^{\prime}, P_{4}^{\prime} P_{-2}^{\prime}$ pass through $P_{\alpha}$, and the lines $P_{1}^{\prime} P_{-4}^{\prime}, P_{2}^{\prime} P_{-3}^{\prime}, P_{3}^{\prime} P_{-2}^{\prime}, P_{4}^{\prime} P_{-1}^{\prime}$ pass through $P_{\beta}$.

If on the other hand we regroup the points into the sets $\left(P_{1}^{\prime} P_{2}^{\prime} P_{-8}^{\prime} P_{-4}^{\prime}\right)$, $\left(P_{-1}^{\prime} P_{-2}^{\prime} P_{3}^{\prime} P_{4}^{\prime}\right)$, we find that these two sets lie in the respective planes

$$
\begin{aligned}
& p_{12} \mu_{1} t_{1}\left(x_{1}+x_{3}\right)-p_{21} \nu_{1}\left(x_{2}+x_{4}\right)=0, \\
& p_{18} \mu_{1} t_{1}\left(x_{1}+x_{3}\right)+p_{21} \nu_{1}\left(x_{2}+x_{4}\right)=0,
\end{aligned}
$$


on the first cone ray $l_{\alpha \beta}$. Moreover these two quadrangles are in perspective from the two points $P_{\gamma}, P_{\delta}$, the four lines $P_{1}^{\prime} P_{3}^{\prime}, P_{2}^{\prime} P_{4}^{\prime}, P_{-3}^{\prime} P_{-1}^{\prime}$, $P_{-4}^{\prime} P_{-2}^{\prime}$ passing through $P_{\gamma}$, and the four lines $P_{1}^{\prime} P_{4}^{\prime}, P_{2}^{\prime} P_{3}^{\prime}, P_{-3}^{\prime} P_{-2}^{\prime}$, $P_{-4}^{\prime} P_{-1}^{\prime}$ passing through $P_{\delta}$.

The equations of the planes osculating the four primary cone cubics at a set of corresponding points $P_{1}, P_{2}, P_{3}, P_{4}$ are, respectively,

$$
\begin{aligned}
& \left(\pi_{1}\right) \quad 3 p_{12}^{2} t^{2} x_{1}-p_{12}^{2} t^{3} x_{2}-p_{21}^{2} x_{8}+3 p_{21}^{2} t x_{4}=0 \\
& \left(\pi_{2}\right) \quad p_{21}^{2} \quad x_{1}-3 p_{21}^{2} t x_{2}-3 p_{12}^{2} t^{2} x_{8}+p_{12}^{2} t^{3} x_{4}=0 \\
& \left(\pi_{8}\right) \quad p_{21}^{2} x_{1}+p_{12}^{2} t^{3} x_{2}-3 p_{12}^{2} t^{2} x_{8}-3 p_{21}^{2} t x_{4}=0 \\
& \left(\pi_{4}\right) \quad 3 p_{12}^{2} t^{2} x_{1}+3 p_{21}^{2} t x_{2}-p_{21}^{2} x_{3}-p_{12}^{2} t^{3} x_{4}=0
\end{aligned}
$$

These four planes pass through a common point $P_{u}$ on $l_{y \delta}$, where

$$
u=\mu t \gamma+\nu \delta .
$$

The equations of the planes osculating the four secondary cone cubics at the points $P_{1}^{\prime}, P_{2}^{\prime}, P_{3}^{\prime}, P_{4}^{\prime}$ are, respectively,

$$
\begin{array}{llllll}
\left(\pi_{1}^{\prime}\right) & p_{12}^{8} & t^{3} x_{1}-p_{12}^{2} p_{21} t^{2} x_{2}+p_{12} p_{21}^{2} t x_{8}-p_{21}^{3} & x_{4}=0 \\
\left(\pi_{2}^{\prime}\right) & p_{12} p_{21}^{2} t x_{1}-p_{21}^{3} & x_{2}+p_{12}^{3} t^{3} x_{3}-p_{12}^{2} p_{21} t^{2} x_{4}=0 \\
\left(\pi_{3}^{\prime}\right) & p_{12} p_{21}^{2} t x_{1}+p_{12}^{2} p_{21} t^{2} x_{2}+p_{12}^{8} t^{3} x_{3}+p_{21}^{3} x_{4}=0 \\
\left(\pi_{4}^{\prime}\right) & p_{12}^{8} \quad t^{3} x_{1}+p_{21}^{8} x_{2}+p_{12} p_{21}^{2} t x_{8}+p_{12}^{2} p_{21} t^{2} x_{4}=0
\end{array}
$$

These four planes pass through a common point $P_{v}$ on $l_{\alpha \beta}$, where

$$
v=p_{21} \alpha+p_{12} t \beta .
$$

The common tangent planes to $Q$ and $Q^{\prime}$ at the points $P_{\alpha}, P_{\beta}$ are given by the equations

$$
\text { (c) } x_{2}+x_{4}=0, \quad(d) \quad x_{1}+x_{3}=0 .
$$

From (17) and (19) we discover that the following sets of planes are collinear:

$$
\left(\pi_{1}^{\prime}, \pi_{3}^{\prime}, d\right), \quad\left(\pi_{2}^{\prime}, \pi_{4}^{\prime}, d\right), \quad\left(\pi_{1}^{\prime}, \pi_{4}^{\prime}, c\right), \quad\left(\pi_{2}^{\prime}, \pi_{3}^{\prime}, c\right) .
$$

The plane determined by the line of intersection of $\pi_{1}^{\prime}, \pi_{2}^{\prime}$ and the line $l_{\alpha \beta}$ has for its equation

$$
p_{12} t\left(x_{1}+x_{3}\right)-p_{21}\left(x_{2}+x_{4}\right)=0,
$$


and the plane determined by the line of intersection of $\pi_{8}^{\prime}, \pi_{4}^{\prime}$ and the line $l_{\alpha \beta}$ has for its equation

$$
p_{12} t\left(x_{1}+x_{3}\right)+p_{21}\left(x_{2}+x_{4}\right)=0
$$

The four planes $(c),(d),(20),(21)$ are all on $l_{\alpha \beta}$, the second pair being harmonic conjugates with respect to the first pair, and vice versa. We may write the equations of the planes osculating the four secondary cone cubics at the points $P_{-1}^{\prime}, P_{-2}^{\prime}, P_{-3}^{\prime}, P_{-4}^{\prime}$ by replacing $t$ with $-t$ in (17). These new planes will pass through the point

$$
v^{\prime}=p_{21} \alpha-p_{12} t \beta
$$

on $l_{\alpha \beta}$, and for this set of planes the following triples are collinear:

$$
\left(\pi_{-1}^{\prime}, \pi_{-3}^{\prime}, d\right), \quad\left(\pi_{-2}^{\prime}, \pi_{-4}^{\prime}, d\right), \quad\left(\pi_{-1}^{\prime}, \pi_{-4}^{\prime}, c\right), \quad\left(\pi_{-2}^{\prime}, \pi_{-3}^{\prime}, c\right) .
$$

The plane determined by the line of intersection of $\pi_{-1}^{\prime}, \pi_{-2}^{\prime}$ and $l_{\alpha \beta}$ is precisely that given by $(21)$, while the plane determined by the line of intersection of $\pi_{-3}^{\prime}, \pi_{-4}^{\prime}$ and $l_{\alpha \beta}$ is given by (20). It follows that in either of two orders,

$$
\left(\pi_{1}^{\prime} \pi_{2}^{\prime} \pi_{3}^{\prime} \pi_{4}^{\prime}\right) \sim\left(\pi_{-3}^{\prime} \pi_{-4}^{\prime} \pi_{-1}^{\prime} \pi_{-2}^{\prime}\right) \quad \text { or } \quad\left(\pi_{1}^{\prime} \pi_{2}^{\prime} \pi_{3}^{\prime} \pi_{4}^{\prime}\right) \sim\left(\pi_{-4}^{\prime} \pi_{-3}^{\prime} \pi_{-2}^{\prime} \pi_{-1}^{\prime}\right),
$$

we can determine a correspondence between these two sets of four planes such that corresponding pairs of lines determine planes on the line $l_{\alpha \beta}$. We find in fact that the lines $\pi_{1}^{\prime} \pi_{3}^{\prime}, \pi_{2}^{\prime} \pi_{4}^{\prime}, \pi_{-1}^{\prime} \pi_{-3}^{\prime}, \pi_{-2}^{\prime} \pi_{-4}^{\prime}$ lie on plane $d$, the lines $\pi_{1}^{\prime} \pi_{4}^{\prime}, \pi_{2}^{\prime} \pi_{3}^{\prime}, \pi_{-1}^{\prime} \pi_{-4}^{\prime}, \pi_{-2}^{\prime}, \pi_{-3}^{\prime}$ lie on plane $c$, the lines $\pi_{1}^{\prime} \pi_{2}^{\prime}$, $\pi_{-3}^{\prime} \pi_{-4}^{\prime}$ lie on plane (20), and the lines $\pi_{3}^{\prime} \pi_{4}^{\prime}, \pi_{-1}^{\prime} \pi_{-2}^{\prime}$ lie on plane (21).

It results that in either of two ways these two sets of four planes are in perspective from a plane. It can be verified that the four lines $\pi_{1}^{\prime} \pi_{-3}^{\prime}, \pi_{2}^{\prime} \pi_{-4}^{\prime}, \pi_{3}^{\prime} \pi_{-1}^{\prime}, \pi_{4}^{\prime} \pi_{-2}^{\prime}$ are coplanar, as are also the four lines $\pi_{1}^{\prime} \pi_{-4}^{\prime}, \pi_{2}^{\prime} \pi_{-3}^{\prime}, \pi_{3}^{\prime} \pi_{-2}^{\prime}, \pi_{4}^{\prime} \pi_{-1}^{\prime}$, the two planes having for their respective equations

$$
\text { (b) } x_{1}-x_{3}=0, \quad \text { (a) } x_{2}-x_{4}=0
$$

Planes $(a),(b)$ are common tangent planes to $Q$ and $Q^{\prime}$ at the respective points $P_{\gamma}, P_{\delta}$, on $l_{\gamma \delta}$.

Without further discussion we note that in either of two ways the two sets of planes $\left(\pi_{1}^{\prime} \pi_{2}^{\prime} \pi_{-3}^{\prime} \pi_{-4}^{\prime}\right),\left(\pi_{3}^{\prime} \pi_{4}^{\prime} \pi_{-1}^{\prime} \pi_{-2}^{\prime}\right)$ are in perspective from a plane, the four lines $\pi_{1}^{\prime} \pi_{3}^{\prime}, \pi_{2}^{\prime} \pi_{4}^{\prime}, \pi_{-3}^{\prime} \pi_{-1}^{\prime}, \pi_{-4}^{\prime} \pi_{-2}^{\prime}$ lying on the plane $(d)$ and the four lines $\pi_{1}^{\prime} \pi_{4}^{\prime}, \pi_{2}^{\prime} \pi_{3}^{\prime}, \pi_{-3}^{\prime} \pi_{-2}^{\prime}, \pi_{-4}^{\prime} \pi_{-1}^{\prime}$ lying 
on the plane $(c)$. Similar perspectivities exist between the two sets of planes $\pi_{1}, \pi_{2}, \pi_{3}, \pi_{4}$, and $\pi_{-1}, \pi_{-2}, \pi_{-8}, \pi_{-4}$. It is not necessary to $\mathrm{dwell}$ further upon this.

We close this part of our discussion by emphasizing the duality which exists between the primary cone cubics, thought of as point loci, and the secondary cone cubics, thought of as the loci of their osculating planes, this duality being of a reciprocal nature.

IV. Other properties OF the cONe cubics. Allied curves

The perspectivities of the cone cubics are by no means their only interesting properties. Without going into unnecessary detal, we will establish in this section a number of theorems which will serve to illustrate the wealth of material awaiting further investigation.

Each of the primary cone cubics determines a linear complex*. Since $C_{1}, \ldots, C_{4}$ are projectively equivalent to the primary flecnode cubic $C_{F^{*}}$, the corresponding four linear complexes will be projectively equivalent to to the complex $L_{1}$. We obtain the equations, in line coordinates, of these four complexes by applying to $\left(L_{1}\right)$ of (4) the transformations of line coordinates which are the consequences of the four transformations $\left(5_{1}\right),\left(5_{2}\right),\left(5_{3}\right),\left(5_{4}\right)$ in point coördinates. We write below, for each of these complexes, its equation in line coördinates together with the pointplane correspondence which it determines. We have

$$
\left(L_{11}\right) 3 \omega_{14}-\omega_{23}=0, u_{1}=3 x_{4}, u_{2}=-x_{3}, u_{3}=x_{2}, u_{4}=-3 x_{1} \text {; }
$$$$
\left(L_{12}\right) \quad \omega_{14}-3 \omega_{23}=0, u_{1}=x_{4}, u_{2}=-3 x_{3}, u_{3}=3 x_{2}, u_{4}=-x_{1} \text {; }
$$$$
\left(L_{13}\right) \quad \omega_{12}+3 \omega_{34}=0, u_{1}=x_{2}, u_{2}=-x_{1}, u_{3}=3 x_{4}, u_{4}=-3 x_{3} \text {; }
$$$$
\left(L_{14}\right) 3 \omega_{12}+\omega_{34}=0, u_{1}=3 x_{2}, u_{2}=-3 x_{1}, u_{3}=x_{4}, u_{4}=-x_{3} \text {. }
$$

The points $A_{1}, \ldots, A_{4}$ which correspond to plane (10) by means of these four linear complexes have for their coördinates, according to (23),

\begin{tabular}{|c|c|c|c|}
\hline & $A_{1}$ & $A_{\mathrm{2}}$ & $A_{3}$ \\
\hline$x_{1}=$ & $t$, & $3 t$ & $3 t$, \\
\hline$x_{2}=$ & 3 , & 1 & 3 , \\
\hline$x_{3}=$ & $3 t$, & & $t$, \\
\hline$=$ & 1, & 3 & 1, \\
\hline
\end{tabular}

The loci $a_{1}, \ldots, a_{4}$ of these four points are of course straight lines, the polar reciprocals of $l_{\alpha \beta}$ with respect to the four complexes $L_{11}, \ldots, L_{14}$.

* The four linear complexes determined by the secondary cone cubics are identical with those determined by the primary cone cubics. 
From (24) and (2) we find that the quadrangles $P_{1} P_{2} P_{3} P_{4}$ and $A_{1} A_{2} A_{3} A_{4}$, both lying in plane (10), are in perspective from the point $P ;(t, 1, t, 1)$, in which this plane is cut by $l_{\gamma \delta}$. In brief, the polar reciprocals of the first cone ray, taken with respect to the linear complexes determined by the primary cone cubics, determine on each plane of the primary pencil associated with $g$ a quadrangle which is in perspective with the quadrangle of the primary cone cubic points of this plane, the locus of the center of perspective being the second cone ray.

A number of similar theorems may be readily obtained by interchanging cone rays and by making use of the secondary, rather than the primary, cone cubic points. We leave these to be enunciated by the reader.

We have already seen that the equation of the general plane on the second cone ray is

$$
x_{1}-x_{3}-t\left(x_{2}-x_{4}\right)=0 .
$$

The points $B_{1}, \ldots, B_{4}$ which correspond to the plane (25) by means of the four complexes $L_{11}, \ldots, L_{14}$ and whose loci $b_{1}, \ldots, b_{4}$ are the polar reciprocals of $l_{\gamma \delta}$, have for their coördinates, by (23),

$$
\begin{array}{rrrrr} 
& B_{1} & B_{2} & B_{3} & B_{4} \\
x_{1}= & t, & 3 t, & 3 t, & t, \\
x_{2}= & 3, & 1, & 3, & 1, \\
x_{3}= & -3 t, & -t, & -t, & -3 t, \\
x_{4}= & -1, & -3, & -1, & -3 .
\end{array}
$$

From (24), (26), and (1) we find that, of the eight lines involved, $a_{1}, a_{2}$, $b_{1}, b_{2}$ lie on $Q^{\prime}$ and $a_{3}, a_{4}, b_{3}, b_{4}$ lie on $Q$. Moreover the points $\left(a_{1} a_{4}\right)$, $\left(a_{2} a_{3}\right),\left(b_{1} b_{4}\right),\left(b_{2} b_{3}\right)$ are on the flecnode tangent $l_{y o}$ and the points $\left(a_{1} a_{3}\right),\left(a_{2} a_{4}\right),\left(b_{1} b_{3}\right),\left(b_{2} b_{4}\right)$ are on the flecnode tangent $l_{z \sigma}$. Summing up these results we find that the four polar reciprocals of the first (second) cone ray, taken with respect to the linear complexes determined by the primary cone cubics associated with $g$, constitute four edges of a tetrahedron whose other two edges are the flecnode tangents, and of these four lines two lie upon each of the complex quadrics.

The four planes (17) are in general distinct, but when $t=p_{21} / p_{12}$, the first and second coincide in the plane whose equation is

$$
x_{1}-x_{2}+x_{3}-x_{4}=0,
$$

and the third and fourth coincide in the plane whose equation is

$$
x_{1}+x_{2}+x_{3}+x_{4}=0 .
$$


When $t=-p_{21} / p_{12}$, the first pair coincide in plane $\left(27_{2}\right)$ and the second pair in plane $\left(27_{1}\right)$. If we take $t= \pm p_{21} i / p_{12}$, the same situation again develops, but this time our planes of coincidence have the equations

$$
\begin{aligned}
& x_{1}+i x_{2}-x_{3}-i x_{4}=0, \\
& x_{1}-i x_{2}-x_{3}+i x_{4}=0 .
\end{aligned}
$$

From (8) we see that the two real planes of this set are on $l_{\alpha \beta}$ and the two imaginary planes are on $l_{\gamma \delta}$. It is interesting to note also that these planes are given by those values of $t$ which give the focal points of the primary cone cubics. From the above considerations it follows that the secondary cone cubics have four osculating planes in common, a real pair intersecting in the first cone ray and an imaginary pair intersecting in the second cone ray. Moreover in each of these planes lie two pairs of secondary cone cubic points. These pairs correspond to two of the four focal points of the primary cone cubics. The four planes (27) may be called the focal planes of the secondary cone cubics.

From (17) and (1) we see that the planes $\pi_{1}^{\prime}, \pi_{2}^{\prime}$ are tangent to $Q$ and the planes $\pi_{3}^{\prime}, \pi_{4}^{\prime}$ are tangent to $Q^{\prime}$. The points of contact have for their coordinates

$$
\begin{aligned}
& \left(C_{1}^{\prime \prime}\right) x_{1}=p_{21}^{3}, \quad x_{2}=p_{12} p_{21}^{2} t, x_{3}=-p_{12}^{2} p_{21} t^{2}, x_{4}=-\quad p_{12}^{3} t^{3} \\
& \left(C_{2}^{\prime \prime}\right) x_{1}=p_{12}^{2} p_{21} t^{2}, x_{2}=p_{12}^{8} t^{8}, x_{3}=-p_{21}^{3}, \quad x_{4}=-p_{12} p_{21}^{2} t \\
& \left(C_{3}^{\prime \prime}\right) x_{1}=p_{12}^{2} p_{21} t^{2}, x_{2}=p_{12} p_{21}^{2} t, x_{3}=-p_{21}^{3}, \quad x_{4}=-p_{12}^{3} t^{3} \\
& \left(C_{4}^{\prime \prime}\right) x_{1}=p_{21}^{3}, \quad x_{2}=p_{12}^{3} t^{3}, x_{3}=-p_{12}^{2} p_{21} t^{2}, x_{4}=-p_{12} p_{21}^{2} t
\end{aligned}
$$

As $t$ varies these four points trace cubics $C_{1}^{\prime \prime}, \ldots, C_{4}^{\prime \prime}$, two lying upon $Q$ and two upon $Q^{\prime}$. We shall call these four curves primary contact cubics.

The planes osculating the four curves $C_{1}^{\prime \prime}, \ldots, C_{4}^{\prime \prime}$ are given by the respective equations

$$
\begin{aligned}
p_{12}^{3} t^{3} x_{1}-3 p_{12}^{2} p_{21} t^{2} x_{2}-3 p_{12} p_{21}^{2} t x_{3}+\quad p_{21}^{3} x_{4} & =0 \\
3 p_{12} p_{21}^{2} t x_{1}-\quad p_{21}^{8} & x_{2}-\quad p_{12}^{3} t^{3} x_{3}+3 p_{12}^{2} p_{21} t^{2} x_{4}=0 \\
3 p_{12} p_{21}^{2} t x_{1}-3 p_{12}^{2} p_{21} t^{2} x_{2}-\quad p_{12}^{3} t^{3} x_{3}+\quad p_{21}^{3} x_{4} & =0 \\
p_{12}^{3} t^{3} x_{1}-\quad p_{21}^{3} & x_{2}-3 p_{12} p_{21}^{2} t x_{3}+3 p_{12}^{2} p_{21} t^{2} x_{4}=0
\end{aligned}
$$

and the points which correspond to these planes by means of $L$ are, respectively, 


$$
\begin{aligned}
& \left(C_{1}^{\prime \prime \prime}\right) x_{1}=3 p_{21}^{2} t, \quad x_{2}=p_{21}^{2}, \quad x_{3}=p_{12}^{2} t^{3}, \quad x_{4}=3 p_{12}^{2} t^{2} \\
& \left(C_{2}^{\prime \prime \prime}\right) x_{1}=p_{12}^{2} t^{3}, \quad x_{2}=3 p_{12}^{2} t^{2}, \quad x_{8}=3 p_{21}^{2} t, x_{4}=p_{21}^{2} \\
& \left(C_{8}^{\prime \prime \prime}\right) x_{1}=p_{12}^{2} t^{3}, \quad x_{2}=p_{21}^{2}, \quad x_{8}=3 p_{21}^{2} t, \quad x_{4}=3 p_{12}^{2} t^{2} \\
& \left(C_{4}^{\prime \prime \prime}\right) x_{1}=3 p_{21}^{2} t, \quad x_{2}=3 p_{12}^{2} t^{2}, \quad x_{3}=p_{12}^{2} t^{3}, x_{4}=p_{21}^{2}
\end{aligned}
$$

Equations (30) define four new cubics $C_{1}^{\prime \prime \prime}, \ldots, C_{4}^{\prime \prime \prime}$, which, by virtue of their relation to the primary contact cubics, we shall speak of as secondary contact cubics.

The equations of the osculating planes of the curves $C_{1}^{\prime \prime \prime}, \ldots, C_{4}^{\prime \prime \prime}$ are, respectively,

$$
\begin{aligned}
& p_{12}^{2} t^{2} x_{1}-p_{12}^{2} t^{3} x_{2}+p_{21}^{2} \quad x_{3}-p_{21}^{2} t x_{4}=0 \\
& p_{21}^{2} \quad x_{1}-p_{21}^{2} t x_{2}+p_{12}^{2} t^{2} x_{3}-p_{12}^{2} t^{3} x_{4}=0 \\
& p_{21}^{2} \quad x_{1}-p_{12}^{2} t^{3} x_{2}+p_{12}^{2} t^{2} x_{8}-p_{21}^{2} t x_{4}=0 \\
& p_{12}^{2} t^{2} x_{1}-p_{21}^{2} t x_{2}+p_{21}^{2} \quad x_{3}-p_{12}^{2} t^{3} x_{4}=0 .
\end{aligned}
$$

A comparison of equations (31) with equations (1) and (2) shows that these planes are tangent to the complex quadrics, the locus of the points of contact being, for the first two, the cubics $C_{1}, C_{2}$ on $Q$, and for the second two the cubics $C_{3}, C_{4}$ on $Q^{\prime}$. Starting with the four primary cone cubics we have thus, after four point transformations of space, returned to these same cubics, and have in the process introduced three other sets of four curves each, all of them cubics. Let us further examine this closed sequence of transformations.

To each point of space there corresponds by means of $L$ a plane, and to this plane there corresponds by means of $L_{11}$ a point. These two compiexes thus determine a point transformation whose analytic expression we proceed to find. The point-plane correspondence determined by $L^{*}$ is given by

$$
u_{1}=p_{12} x_{3}, \quad u_{2}=-p_{21} x_{4}, \quad u_{3}=-p_{12} x_{1}, \quad u_{4}=p_{21} x_{2} .
$$

From (32) and the first of equations (23) the equations of this point transformation are easily obtained. They are

$$
\bar{x}_{1}=-p_{21} x_{2}, \quad \bar{x}_{2}=-3 p_{12} x_{1}, \quad \bar{x}_{3}=3 p_{21} x_{4}, \quad \bar{x}_{4}=p_{12} x_{3} .
$$

Associating thus with $L$ each of the complexes of (23) in turn, we obtain four such point transformations. They are

*Proj. Dif. Geom., p. 206. 


$$
\begin{aligned}
& \left(L, L_{11}\right) \bar{x}_{1}=-p_{21} x_{2}, \bar{x}_{2}=-3 p_{12} x_{1}, \bar{x}_{3}=3 p_{21} x_{4}, \bar{x}_{4}=p_{12} x_{3} ; \\
& \left(L, L_{12}\right) \bar{x}_{1}=-3 p_{21} x_{2}, \bar{x}_{2}=-p_{12} x_{1}, \bar{x}_{3}=p_{21} x_{4}, \bar{x}_{4}=3 p_{12} x_{3} ; \\
& \left(L, L_{13}\right) \bar{x}_{1}=-3 p_{21} x_{4}, \bar{x}_{2}=-3 p_{12} x_{3}, \bar{x}_{3}=p_{21} x_{2}, \bar{x}_{4}=p_{12} x_{1} ; \\
& \left(L, L_{14}\right) \bar{x}_{1}=-p_{21} x_{4}, \bar{x}_{2}=-p_{12} x_{3}, \bar{x}_{3}=3 p_{21} x_{2}, \bar{x}_{4}=3 p_{12} x_{1} .
\end{aligned}
$$

We note in passing that these transformations are each of period two and hence that the point correspondences determined by them are reciprocal.

Since each quadric determines a $(1,1)$ correspondence between the points and planes of space, we may set up a point transformation by making use of a linear complex and a quadric. For to each point there corresponds its polar plane by means of the complex and to this plane there corresponds its pole with respect to the quadric. Making use of this notion we define a second set of four point transformations, their expressions being

$$
\begin{array}{lll}
\left(L_{11}, Q\right) & \bar{x}_{1}=-3 x_{1}, \bar{x}_{2}=-x_{2}, \bar{x}_{3}=x_{3}, \bar{x}_{4}=3 x_{4} \\
\left(L_{12}, Q\right) & \bar{x}_{1}=-x_{1}, \bar{x}_{2}=-3 x_{2}, \bar{x}_{3}=3 x_{3}, \bar{x}_{4}=x_{4} \\
\left(L_{13}, Q^{\prime}\right) & \bar{x}_{1}=-x_{1}, \bar{x}_{2}=x_{2}, \bar{x}_{3}=3 x_{3}, \bar{x}_{4}=-3 x_{4} \\
\left(L_{14}, Q^{\prime}\right) & \bar{x}_{1}=-3 x_{1}, \bar{x}_{2}=3 x_{2}, \bar{x}_{3}=x_{3}, \bar{x}_{4}=-x_{4} .
\end{array}
$$

The eight transformations of (33) and (34), taken four at a time in the proper order, carry the four primary cone cubics through their four-phase cycle. Symbolically we have

$$
\begin{aligned}
& \left(L_{12}, Q\right)\left[\left(L, L_{18}\right)\left[\left(L_{11}, Q\right)\left[\left(L, L_{11}\right) C_{1}=C_{1}^{\prime}\right]=C_{1}^{\prime \prime}\right]=C_{1}^{\prime \prime \prime}\right]=C_{1}, \\
& \left(L_{11}, Q\right)\left[\left(L, L_{11}\right)\left[\left(L_{12}, Q\right)\left[\left(L, L_{12}\right) C_{2}=C_{2}^{\prime}\right]=C_{2}^{\prime \prime}\right]=C_{2}^{\prime \prime \prime}\right]=C_{2}, \\
& \left(L_{14}, Q^{\prime}\right)\left[\left(L, L_{14}\right)\left[\left(L_{13}, Q^{\prime}\right)\left[\left(L, L_{13}\right) C_{3}=C_{3}^{\prime}\right]=-C_{3}^{\prime \prime}\right]=C_{3}^{\prime \prime \prime}\right]=C_{3}, \\
& \left(L_{13}, Q^{\prime}\right)\left[\left(L, L_{13}\right)\left[\left(L_{14}, Q^{\prime}\right)\left[\left(L, L_{14}\right) C_{4}=C_{1}^{\prime}\right]=C_{4}^{\prime \prime}\right]=C_{4}^{\prime \prime \prime}\right]=C_{4} .
\end{aligned}
$$

It has been noted that the primary and secondary cone cubics determine the same set $L_{11}, L_{12}, L_{13}, L_{14}$ of linear complexes. Without difficulty it can be shown that the primary and secondary contact cubics determine the same four linear complexes, but in the order $L_{12}, L_{11}, L_{14}, L_{13}$. Many additional properties of these curves might be developed. It will be sufficient however to summarize the results of the last few paragraphs and then to state without proof a number of additional theorems whose truth can be demonstrated with the material at hand.

We find that associated with each line element $g$ of the general ruled surface there are sixteen projectively equivalent space cubics so related in cyclically ordered sets of four each that the points of the curves in any set lie upon the osculating planes of the curves of the preceding set. 
The curves of two of the four sets lie upon the complex quadrics associated with $g$, two from each set on each quadric, while the osculating planes of the curves of the other two sets are tangent to these quadrics.

The four linear complexes determined by the curves of any set are distinct, but any two sets determine the same four complexes.

Among the theorems whose proofs are left to the reader we have the following:

1. The primary (secondary) contact cubics are the loci of the poles of the osculating planes of the secondary (primary) cone cubics taken with respect to the complex quadrics, and conversely.

2. The cubics $C_{j}^{k}(j=1, \cdots, 4 ; k=0, \cdots, 3)$ belong to the complexes $L_{1 i}, C_{j}$ and $C_{j}^{\prime}$ belonging to $L_{1 i}(i=j)$, and $C_{j}^{\prime \prime}$ and $C_{j}^{\prime \prime \prime}$ belonging to $L_{1 i}$ where $i=j-(-1)^{j}$.

3. The ruled surfaces determined by the point correspondences set up between the pairs of cubics $C_{j}^{k}, C_{j}^{k+1}(j=1, \cdots, 4 ; k=0, \cdots, 3)$, by the parameter $t$, belong to one of the four complexes $L_{1 i}$, those determined by $C_{j}, C_{j}^{\prime}$ and $C_{j}^{\prime}, C_{j}^{\prime \prime}$ belonging to $L_{1 i}(i=j)$, and those determined by $C_{j}^{\prime \prime}, C_{j}^{\prime \prime \prime}$ and $C_{j}^{\prime \prime \prime}, C_{j}$ belonging to $L_{1 i}$ where $i=j-(-1)^{j}$.

4. Each of the cubics $C_{j}^{k}$ generates a surface as the line element $g$ with which it is associated varies over the ruled surface $S$. Of these surfaces the eight generated by the primary cone cubics and the secondary contact cubics are projectively equivalent, as are also the eight generated by the secondary cone cubics and the primary contact cubics.

5. Four of the sixteen surfaces $S_{j}^{k}$ generated by the cubics $C_{j}^{k}$ are tangent to the ruled surface $S, S_{1}^{\prime}$ and $S_{1}^{\prime \prime}$ being tangent to $S$ along the branch $C_{y}$ of the flecnode curve, and $S_{1}$ and $S_{1}^{\prime \prime \prime}$ tangent to $S$ along the branch $C_{z}$ of this curve. Of the remaining twelve surfaces, $S_{2}$ and $S_{2}^{\prime \prime \prime}$ cut $S$ along $C_{y}$, $S_{2}^{\prime}$ and $S_{2}^{\prime \prime}$ cut $S$ along $C_{z}$, while $S_{3}, S_{4}^{\prime}, S_{4}^{\prime \prime}$, and $S_{3}^{\prime \prime \prime}$ cut $S$ along both $C_{y}$ and $C_{z}$.

6. The point correspondence existing between each pair of primary cone cubics $C_{j}$ determines a ruled surface on which this pair of cubics are directrix curves. Of the six surfaces thus determined, those given by $C_{1}, C_{2}$ and by $C_{3}, C_{4}$ are the two complex quadrics. The remaining four are cubic cones with vertices at the points $P_{\alpha}, P_{\beta}$. Their equations, in the system of coördinates here employed, are

$$
\begin{aligned}
& \left(C_{1}, C_{3}\right) \quad p_{12}^{2} x_{2}\left(x_{1}+x_{3}\right)^{2}+p_{21}^{2} x_{4}\left(x_{2}+x_{4}\right)^{2}=0, \text { vertex at } P_{\alpha} \\
& \left(C_{1}, C_{4}\right) \quad p_{12}^{2} x_{1}\left(x_{1}+x_{3}\right)^{2}+p_{21}^{2} x_{3}\left(x_{2}+x_{4}\right)^{2}=0, \quad " \quad " P_{\beta} \text {; } \\
& \left(C_{2}, C_{3}\right) \quad p_{12}^{2} x_{3}\left(x_{1}+x_{3}\right)^{2}+p_{21}^{2} x_{1}\left(x_{2}+x_{4}\right)^{2}=0, \quad \text { " " } P_{\beta} \text {; } \\
& \left(C_{2}, C_{4}\right) \quad p_{12}^{2} x_{4}\left(x_{1}+x_{8}\right)^{2}+p_{21}^{2} x_{2}\left(x_{2}+x_{4}\right)^{2}=0, \quad " \quad " P_{\alpha} \text {. }
\end{aligned}
$$


7. The primary contact cubics determine among themselves six ruled surfaces two of which are the complex quadrics, and the remaining four, cubic cones. The equations of the latter four are

$$
\begin{array}{llll}
\left(C_{1}^{\prime \prime}, C_{3}^{\prime \prime}\right) & x_{4}\left(x_{1}-x_{3}\right)^{2}+x_{2}\left(x_{2}-x_{4}\right)^{2}=0, & \text { vertex } & \text { at } P_{\gamma} \\
\left(C_{1}^{\prime \prime}, C_{4}^{\prime \prime}\right) & x_{3}\left(x_{1}-x_{3}\right)^{2}+x_{1}\left(x_{2}-x_{4}\right)^{2}=0, \quad \text { " } & P_{\delta} \\
\left(C_{2}^{\prime \prime}, C_{3}^{\prime \prime}\right) & x_{1}\left(x_{1}-x_{3}\right)^{2}+x_{3}\left(x_{2}-x_{4}\right)^{2}=0, \quad \text { " } P_{\delta} \\
\left(C_{2}^{\prime \prime}, C_{4}^{\prime \prime}\right) & x_{2}\left(x_{1}-x_{3}\right)^{2}+x_{4}\left(x_{2}-x_{4}\right)^{2}=0, \quad \text { " } & P_{\gamma} .
\end{array}
$$

No attempt has been made in this paper to investigate the properties of the loci which the points, lines, curves and surfaces here discussed will generate when the line-element $g$ with which they are associated varies over the surface $S$. Nor has it been thought advisable to consider the results of imposing upon $S$ any special conditions. The methods of attacking all of these problems are available and their solutions, while requiring some ingenuity, should involve no great difficulties.

UNIVERSITY OF WASHINGTON,

Seattle, Wash. 